International Journal of Pure and Applied Mathematics

Volume 87 No. 1 2013, 151-164

ISSN: 1311-8080 (printed version); ISSN: 1314-3395 (on-line version)

url: http://www.ijpam.eu

doi: http://dx.doi.org/10.12732/ijpam.v87i1.9

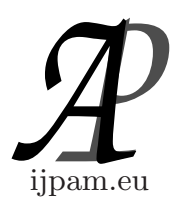

\title{
REGULARITY IN SEMIGROUPS OF TRANSFORMATIONS WITH INVARIANT SETS
}

\author{
Wanida Choomanee ${ }^{1}$, Preeyanuch Honyam ${ }^{2}$, Jintana Sanwong $3 \S$ \\ 1,2,3 Department of Mathematics \\ Faculty of Science \\ Chiang Mai University \\ Chiangmai, 50200, THAILAND
}

\begin{abstract}
Let $T(X)$ be the semigroup of all transformations on a set $X$. For a fixed nonempty subset $Y$ of $X$, let

$$
S(X, Y)=\{\alpha \in T(X): Y \alpha \subseteq Y\} .
$$

Then $S(X, Y)$ is a semigroup of total transformations on $X$ which leave a subset $Y$ of $X$ invariant. In this paper, we characterize left regular, right regular and intra-regular elements of $S(X, Y)$ and consider the relationships between these elements. Moreover, we count the number of left regular elements of $S(X, Y)$ when $X$ is a finite set.
\end{abstract}

AMS Subject Classification: 20M20

Key Words: transformation semigroups, left regular element, right regular element, intra-regular element

\section{Introduction}

Let $X$ be a nonempty set and let $T(X)$ denote the set of all transformations from $X$ into itself. Then $T(X)$ is a semigroup under the composition of maps and it is called the full transformation semigroup on $X$.

It is known that $T(X)$ is a regular semigroup, that is, for every $\alpha \in T(X)$, $\alpha=\alpha \beta \alpha$ for some $\beta \in T(X)$.

Received: June 4, 2013

(c) 2013 Academic Publications, Ltd. url: www.acadpubl.eu

$\S$ Correspondence author 
In this paper, we study the subsemigroup of $T(X)$, namely $S(X, Y)$, defined by

$$
S(X, Y)=\{\alpha \in T(X): Y \alpha \subseteq Y\}
$$

where $\emptyset \neq Y \subseteq X$. Then $S(X, Y)$ is a semigroup of total transformations on $X$ which leave a subset $Y$ of $X$ invariant. This semigroup was first introduced and studied by K.D. Magill [2] in 1966. In fact, if $Y=X$, then $S(X, Y)=T(X)$. So we may regard $S(X, Y)$ as a generalization of $T(X)$.

In 2005, S. Nenthein, P. Youngkhong and Y. Kemprasit [4] showed that $S(X, Y)$ is a regular semigroup if and only if $X=Y$ or $Y$ contains exactly one element, and $\operatorname{Reg} S(X, Y)=\{\alpha \in S(X, Y): X \alpha \cap Y=Y \alpha\}$ is the set of all regular elements of $S(X, Y)$. Moreover, they count the number of regular elements of $S(X, Y)$ when $X$ is a finite set.

In 2011, P. Honyam and J. Sanwong [3] described Green's relations and ideals on $S(X, Y)$. Here, in Section 3, we characterize left regular, right regular and intra-regular elements on $S(X, Y)$ and consider the relationships between these elements. In Section 4, we count the number of left regular elements of $S(X, Y)$ when $X$ is a finite set.

\section{Preliminaries}

In this section, we list some known results, definitions and notations that will be used throughout this paper.

Let $X$ be a set and $Y$ a nonempty subset of $X$. We defined

$$
S(X, Y)=\{\alpha \in T(X): Y \alpha \subseteq Y\} .
$$

For $\alpha, \beta \in S(X, Y)$, we have $Y \alpha \subseteq Y$ and $Y \beta \subseteq Y$. Then $Y \alpha \beta \subseteq Y \beta \subseteq Y$, so $\alpha \beta \in S(X, Y)$. Therefore, $S(X, Y)$ is a subsemigroup of $T(X)$. From [4], we have $S(X, Y)$ is regular if and only if $X=Y$ or $|Y|=1$. That is, in general $S(X, Y)$ is not a regular semigroup. Let

$$
\operatorname{Reg} S(X, Y)=\{\alpha \in S(X, Y): X \alpha \cap Y=Y \alpha\} .
$$

Then Reg $S(X, Y)$ is the set of all regular elements of $S(X, Y)$.

As in A. H. Clifford and G. B. Preston [1], we shall use the notation

$$
\alpha=\left(\begin{array}{c}
X_{i} \\
a_{i}
\end{array}\right)
$$


to mean that $\alpha \in T(X)$ and take as understood that the subscript $i$ belongs to some (unmentioned) index set $I$, the abbreviation $\left\{a_{i}\right\}$ denotes $\left\{a_{i}: i \in I\right\}$, and that $X \alpha=\left\{a_{i}\right\}$ and $a_{i} \alpha^{-1}=X_{i}$ for all $i$.

With the above notation, for any $\alpha \in S(X, Y)$ we can write

$$
\alpha=\left(\begin{array}{ccc}
A_{i} & B_{j} & C_{k} \\
a_{i} & b_{j} & c_{k}
\end{array}\right),
$$

where $A_{i} \cap Y \neq \emptyset, B_{j}, C_{k} \subseteq X \backslash Y,\left\{a_{i}\right\} \subseteq Y,\left\{b_{j}\right\} \subseteq Y \backslash\left\{a_{i}\right\}$ and $\left\{c_{k}\right\} \subseteq X \backslash Y$. Here, $I$ is a nonempty set, but $J$ or $K$ can be empty. For examples: If $\alpha \in$ $\operatorname{Reg} S(X, Y)$, then $J$ is an empty set. And if $\alpha \in S(X, Y) \backslash \operatorname{Reg} S(X, Y)$, then both $I$ and $J$ are nonempty but $K$ can be an empty set.

We note that for any $\alpha \in S(X, Y)$, the symbol $\pi_{\alpha}$ denotes the decomposition of $X$ induced by the map $\alpha$, namely $\pi_{\alpha}=\left\{x \alpha^{-1}: x \in X \alpha\right\}$. For a nonempty subset $Z$ of $X$, we denote $\pi_{\alpha}(Z)$ by $\pi_{\alpha}(Z)=\left\{x \alpha^{-1}: x \in X \alpha \cap Z\right\}$. Thus $\pi_{\alpha}(Y)=\left\{y \alpha^{-1}: y \in X \alpha \cap Y\right\}$.

The following theorem is given by S. Nenthein, P. Youngkhong and Y. Kemprasit [4].

Theorem 2.1. The following statements hold for the semigroup $S(X, Y)$.

(1) For $\alpha \in S(X, Y), \alpha$ is a regular element of $S(X, Y)$ if and only if $X \alpha \cap Y=Y \alpha$.

(2) $S(X, Y)$ is regular if and only if either $Y=X$ or $|Y|=1$.

Green's relations on $S(X, Y)$ are given by P. Honyam and J. Sanwong [3], which are needed in characterizing left regular, right regular and intra-regular elements on $S(X, Y)$.

Lemma 2.2. Let $\alpha, \beta \in S(X, Y)$. Then:

(1) $\alpha \mathcal{L} \beta$ if and only if $X \alpha=X \beta$ and $Y \alpha=Y \beta$.

(2) $\alpha \mathcal{R} \beta$ if and only if $\pi_{\alpha}=\pi_{\beta}$ and $\pi_{\alpha}(Y)=\pi_{\beta}(Y)$.

(3) $\alpha \mathcal{J} \beta$ if and only if $|X \alpha|=|X \beta|,|Y \alpha|=|Y \beta|$ and $|X \alpha \backslash Y|=|X \beta \backslash Y|$.

\section{Left Regular, Right Regular and Intra-Regular Elements}

In this section, we give necessary and sufficient conditions for elements in $S(X, Y)$ to be left regular, right regular and intra-regular. We first recall that: 
an element $\alpha \in S(X, Y)$ is left regular [right regular] if $\alpha=\beta \alpha^{2}\left[\alpha=\alpha^{2} \beta\right]$ for some $\beta \in S(X, Y)$ and it is intra-regular if $\alpha=\lambda \alpha^{2} \mu$ for some $\lambda, \mu \in S(X, Y)$. Since the identity map $1_{X} \in S(X, Y)$, it follows that $S(X, Y)^{1}=S(X, Y)$. For each $\alpha \in S(X, Y)$, we denote $\mathcal{L}$-class, $\mathcal{R}$-class and $\mathcal{J}$-class containing $\alpha$ by $L_{\alpha}, R_{\alpha}$ and $J_{\alpha}$ respectively.

Theorem 3.1. Let $\alpha \in S(X, Y)$. Then the following statements are equivalent:

(1) $\alpha$ is left regular.

(2) $X \alpha=X \alpha^{2}$ and $Y \alpha=Y \alpha^{2}$.

(3) $\alpha^{2} \in L_{\alpha}$.

Proof. It is clear that $(2) \Leftrightarrow(3)$ by Lemma 2.2(1). If $\alpha$ is left regular, then $\alpha=\beta \alpha^{2}$ for some $\beta \in S(X, Y)$ and we have $\alpha^{2}=\alpha \cdot \alpha$, so $\alpha \mathcal{L} \alpha^{2}$ and that $\alpha^{2} \in L_{\alpha}$. Conversely, if $\alpha^{2} \in L_{\alpha}$, then $\alpha=\beta \alpha^{2}$ for some $\beta \in S(X, Y)$. So $\alpha$ is left regular.

Similar to Theorem 3.1, by using Lemma 2.2(2), we obtain the following theorem.

Theorem 3.2. Let $\alpha \in S(X, Y)$. Then the following statements are equivalent:

(1) $\alpha$ is right regular.

(2) $\pi_{\alpha}=\pi_{\alpha^{2}}$ and $\pi_{\alpha}(Y)=\pi_{\alpha^{2}}(Y)$.

(3) $\alpha^{2} \in R_{\alpha}$.

In general, there is no relationships between left regular elements and right regular elements as shown in the following example.

Example 1. Let $X$ be the set of all natural numbers.

(1) Let $Y=\{1,2,3,4\}$ and define $\alpha$ by

$$
n \alpha= \begin{cases}n & , n \in\{1,2\} \\ 4 & , n=3 \\ 3 & , n \in\{4,5\} \\ n-1 & , \text { otherwise }\end{cases}
$$


Then $\alpha \in S(X, Y)$ and

$$
n \alpha^{2}= \begin{cases}n & , n \in Y \\ 4 & , n=5 \\ 3 & , n=6 \\ n-2 & , \text { otherwise }\end{cases}
$$

Thus $X \alpha=X \alpha^{2}$ is the set of all natural numbers and $Y \alpha=\{1,2,3,4\}=Y \alpha^{2}$. So $\alpha$ is left regular. But $\alpha$ is not right regular, since

$$
\pi_{\alpha}(Y)=\{\{1\},\{2\},\{3\},\{4,5\}\} \neq\{\{1\},\{2\},\{3,6\},\{4,5\}\}=\pi_{\alpha^{2}}(Y) .
$$

(2) Let $Y$ the set of all positive even integers, and let $\alpha$ be defined by $n \alpha=n+2$ for all $n \in X$. Then $\alpha \in S(X, Y)$ and $n \alpha^{2}=n+4$ for all $n \in X$. Thus $\pi_{\alpha}(Y)=\{\{2 n\}\}=\pi_{\alpha^{2}}(Y)$ and $\pi_{\alpha}=\{\{n\}\}=\pi_{\alpha^{2}}$. So $\alpha$ is right regular. But $\alpha$ is not left regular, since $Y \alpha=\{2 n+2\} \neq\{2 n+4\}=Y \alpha^{2}$.

For convenience, from now on if $\alpha \in S(X, Y)$, the notations $Y^{\prime}$ and $X^{\prime}$ are for $Y \alpha$ and $X \alpha \backslash Y \alpha$ respectively. To prove that $\alpha \in S(X, Y)$ is left regular if and only if $\alpha$ is right regular when $X \alpha$ is finite, we begin with the following three lemmas.

Lemma 3.3. Let $\alpha \in S(X, Y)$. If $X \alpha=X \alpha^{2}$ is finite and $Y \alpha=Y \alpha^{2}$, then

(1) $(X \backslash Y) \alpha \subseteq(X \backslash Y) \cup Y^{\prime}$;

(2) $X^{\prime} \subseteq X \backslash Y^{\prime} \alpha^{-1}$;

(3) $\left|y^{\prime} \alpha^{-1} \cap Y^{\prime}\right|=1$ for all $y^{\prime} \in Y^{\prime}$;

(4) $\left|x^{\prime} \alpha^{-1} \cap X^{\prime}\right|=1$ for all $x^{\prime} \in X^{\prime}$.

Proof. Assume that $X \alpha=X \alpha^{2}$ is finite and $Y \alpha=Y \alpha^{2}$. So we can write

$$
\alpha=\left(\begin{array}{ccccccccc}
A_{1} & \ldots & A_{n} & B_{1} & \ldots & B_{m} & C_{1} & \ldots & C_{t} \\
a_{1} & \ldots & a_{n} & b_{1} & \ldots & b_{m} & c_{1} & \ldots & c_{t}
\end{array}\right)
$$

where $A_{i} \cap Y \neq \emptyset, B_{j}, C_{k} \subseteq X \backslash Y ; a_{i}, b_{j} \in Y, c_{k} \in X \backslash Y$ for all $i=1, \ldots, n, j=$ $1, \ldots, m$ and $k=1, \ldots, t$. Then $Y^{\prime}=\left\{a_{1}, \ldots, a_{n}\right\}$ and $X^{\prime}=\left\{b_{1}, \ldots, b_{m}, c_{1}, \ldots, c_{t}\right\}$.

(1) We prove that $J=\emptyset$ where $J=\{1, \ldots, m\}$. Suppose that there is $j \in J$ such that $B_{j} \alpha=\left\{b_{j}\right\}$ where $b_{j} \in Y$. Then $B_{j} \alpha^{2}=\left(B_{j} \alpha\right) \alpha=\left\{b_{j}\right\} \alpha$. Assume 
that $b_{j} \in A_{i_{0}}$ for some $i_{0}$. Thus $B_{j} \alpha^{2}=\left\{b_{j}\right\} \alpha=\left\{a_{i_{0}}\right\}$. Since $Y \alpha^{2}=Y \alpha=$ $\left\{a_{1}, \ldots, a_{n}\right\}$, there is $A_{k}$ such that $A_{k} \alpha^{2}=\left\{a_{i_{0}}\right\}$. That is $A_{k} \alpha^{2}=B_{j} \alpha^{2}$. But $A_{k} \alpha \neq B_{j} \alpha$, so $\left|X \alpha^{2}\right| \leq n+(m-1)+t<|X \alpha|$, which implies that $X \alpha^{2} \subsetneq X \alpha$, a contradiction. Thus $J=\emptyset$. So we can write

$$
\alpha=\left(\begin{array}{cccccc}
A_{1} & \ldots & A_{n} & C_{1} & \ldots & C_{t} \\
a_{1} & \ldots & a_{n} & c_{1} & \ldots & c_{t}
\end{array}\right)
$$

where $A_{i} \cap Y \neq \emptyset, C_{k} \subseteq X \backslash Y ; a_{i} \in Y, c_{k} \in X \backslash Y$ for all $i=1, \ldots, n$ and $k=1, \ldots, t$. Then $Y^{\prime}=\left\{a_{1}, \ldots, a_{n}\right\}$ and $X^{\prime}=\left\{c_{1}, \ldots, c_{t}\right\}$. Therefore, $(X \backslash Y) \alpha \subseteq$ $(X \backslash Y) \cup Y^{\prime}$.

(2) Now, we have $X^{\prime}=\left\{c_{1}, \ldots, c_{t}\right\} \subseteq X \backslash Y$ and $X \backslash Y^{\prime} \alpha^{-1}=C_{1} \cup \ldots \cup C_{t}$. Suppose that there is $c_{p} \in X^{\prime} \cap Y^{\prime} \alpha^{-1}$. So $c_{p} \in A_{i_{0}}$ for some $i_{0}$. Then $C_{p} \alpha^{2}=$ $\left\{a_{i_{0}}\right\}$. Since $Y \alpha^{2}=Y \alpha$, there is $A_{q}$ such that $A_{q} \alpha^{2}=\left\{a_{i_{0}}\right\}$, so we get $C_{p} \alpha^{2}=$ $\left\{a_{i_{0}}\right\}=A_{q} \alpha^{2}$, thus $\left|X \alpha^{2}\right| \leq n+(t-1)<|X \alpha|$ which implies that $X \alpha^{2} \subsetneq X \alpha$, a contradiction. Therefore, $X^{\prime} \cap Y^{\prime} \alpha^{-1}=\emptyset$, hence $X^{\prime} \subseteq X \backslash Y^{\prime} \alpha^{-1}$.

(3) Assume that $\left|y^{\prime} \alpha^{-1} \cap Y^{\prime}\right| \neq 1$ for some $y^{\prime} \in Y^{\prime}$. Suppose that there are $a_{i}$ and $a_{j}$ both belong to $A_{l}$ for some $l \in\{1, \ldots, n\}$ where $a_{i} \neq a_{j} \in Y^{\prime}$. Then $A_{i} \alpha^{2}=\left\{a_{l}\right\}=A_{j} \alpha^{2}$. Thus $A_{i} \alpha^{2}=A_{j} \alpha^{2}$, so $\left|Y \alpha^{2}\right| \leq n-1$ where $|Y \alpha|=n$, a contradiction. So $a_{i}$ and $a_{j}$ belong to different $A_{l}$ for all $i \neq j$. Since $\left|Y^{\prime}\right|=n$ is finite, we obtain $Y^{\prime} \cap A_{l} \neq \emptyset$ for all $l$ and that $\left|y^{\prime} \alpha^{-1} \cap Y^{\prime}\right|=1$ for all $y^{\prime} \in Y^{\prime}$.

(4) Assume that $\left|x^{\prime} \alpha^{-1} \cap X^{\prime}\right| \neq 1$ for some $x^{\prime} \in X^{\prime}$. Suppose that there are $c_{u}$ and $c_{v} \in C_{k}$ for some $k \in\{1, \ldots, t\}$ where $c_{u} \neq c_{v} \in X^{\prime}$. Then $C_{u} \alpha^{2}=$ $\left\{c_{k}\right\}=C_{v} \alpha^{2}$ and thus $C_{u} \alpha^{2}=C_{v} \alpha^{2}$, a contradiction, since $X \alpha^{2}=X \alpha$. So $c_{u}$ and $c_{v}$ belong to different $C_{k}$ for all $u \neq v$. Since $\left|X^{\prime}\right|=t$ is finite, it follows that $X^{\prime} \cap C_{k} \neq \emptyset$ for all $k$. Therefore, $\left|x^{\prime} \alpha^{-1} \cap X^{\prime}\right|=1$ for all $x^{\prime} \in X^{\prime}$.

Lemma 3.4. Let $\alpha \in S(X, Y)$. If $\pi_{\alpha}=\pi_{\alpha^{2}}$ is finite and $\pi_{\alpha}(Y)=\pi_{\alpha^{2}}(Y)$ then, $\pi_{\alpha}(Y \alpha)=\pi_{\alpha^{2}}(Y \alpha)$.

Proof. Assume that $\pi_{\alpha}=\pi_{\alpha^{2}}$ is finite and $\pi_{\alpha}(Y)=\pi_{\alpha^{2}}(Y)$. We have $\pi_{\alpha}(Y \alpha)=\left\{y \alpha^{-1}: y \in X \alpha \cap Y \alpha\right\}$ and $\pi_{\alpha^{2}}(Y \alpha)=\left\{y\left(\alpha^{2}\right)^{-1}: y \in X \alpha^{2} \cap Y \alpha\right\}$. Since $\pi_{\alpha}$ is finite, we can write

$$
\alpha=\left(\begin{array}{ccccccccc}
A_{1} & \ldots & A_{n} & B_{1} & \ldots & B_{m} & C_{1} & \ldots & C_{t} \\
a_{1} & \ldots & a_{n} & b_{1} & \ldots & b_{m} & c_{1} & \ldots & c_{t}
\end{array}\right)
$$

where $A_{i} \cap Y \neq \emptyset, B_{j}, C_{k} \subseteq X \backslash Y ; a_{i}, b_{j} \in Y, c_{k} \in X \backslash Y$ for all $i=1, \ldots, n$, $j=1, \ldots, m$ and $k=1, \ldots, t$. Since $\pi_{\alpha}=\pi_{\alpha^{2}}$ and $\pi_{\alpha}(Y)=\pi_{\alpha^{2}}(Y)$, we have

$$
\alpha^{2}=\left(\begin{array}{ccccccccc}
A_{1} & \ldots & A_{n} & B_{1} & \ldots & B_{m} & C_{1} & \ldots & C_{t} \\
a_{1}^{\prime} & \ldots & a_{n}^{\prime} & b_{1}^{\prime} & \ldots & b_{m}^{\prime} & c_{1}^{\prime} & \ldots & c_{t}^{\prime}
\end{array}\right)
$$


where $a_{i}^{\prime}, b_{j}^{\prime} \in Y$ and $c_{k}^{\prime} \in X \backslash Y$. So $Y \alpha^{2}=\left\{a_{1}^{\prime}, \ldots, a_{n}^{\prime}\right\}$, while $Y \alpha=\left\{a_{1}, \ldots, a_{n}\right\}$. Since $Y \alpha \subseteq Y$, we obtain $Y \alpha^{2} \subseteq Y \alpha$. So $\left\{a_{1}^{\prime}, \ldots, a_{n}^{\prime}\right\} \subseteq\left\{a_{1}, \ldots, a_{n}\right\}$. Since $Y \alpha$ is finite, we have $\left\{a_{1}^{\prime}, \ldots, a_{n}^{\prime}\right\}=\left\{a_{1}, \ldots, a_{n}\right\}$. Thus $X \alpha \cap Y \alpha=X \alpha^{2} \cap Y \alpha$. Therefore, $\pi_{\alpha}(Y \alpha)=\left\{A_{1}, \ldots, A_{n}\right\}=\pi_{\alpha^{2}}(Y \alpha)$.

Lemma 3.5. Let $\alpha \in S(X, Y)$. If $\pi_{\alpha}=\pi_{\alpha^{2}}$ is finite and $\pi_{\alpha}(Y)=\pi_{\alpha^{2}}(Y)$, then

(1) $(X \backslash Y) \alpha \subseteq(X \backslash Y) \cup Y^{\prime}$;

(2) $X^{\prime} \subseteq X \backslash Y^{\prime} \alpha^{-1}$;

(3) $\left|y^{\prime} \alpha^{-1} \cap Y^{\prime}\right|=1$ for all $y^{\prime} \in Y^{\prime}$;

(4) $\left|x^{\prime} \alpha^{-1} \cap X^{\prime}\right|=1$ for all $x^{\prime} \in X^{\prime}$.

Proof. Since $\pi_{\alpha}$ is finite, so we can write

$$
\alpha=\left(\begin{array}{ccccccccc}
A_{1} & \ldots & A_{n} & B_{1} & \ldots & B_{m} & C_{1} & \ldots & C_{t} \\
a_{1} & \ldots & a_{n} & b_{1} & \ldots & b_{m} & c_{1} & \ldots & c_{t}
\end{array}\right)
$$

where $A_{i} \cap Y \neq \emptyset, B_{j}, C_{k} \subseteq X \backslash Y ; a_{i}, b_{j} \in Y, c_{k} \in X \backslash Y$ for all $i=1, \ldots, n, j=$ $1, \ldots, m$ and $k=1, \ldots, t$. Then $Y^{\prime}=\left\{a_{1}, \ldots, a_{n}\right\}$ and $X^{\prime}=\left\{b_{1}, \ldots, b_{m}, c_{1}, \ldots, c_{t}\right\}$. Since $\pi_{\alpha}=\pi_{\alpha^{2}}$ is finite and $\pi_{\alpha}(Y)=\pi_{\alpha^{2}}(Y)$, we have by Lemma 3.4 that $\pi_{\alpha^{2}}(Y \alpha)=\pi_{\alpha}(Y \alpha)=\left\{A_{1}, \ldots\right.$ $\left.A_{n}\right\}$.

(1) Suppose that there is $j \in J$ such that $B_{j} \alpha=\left\{b_{j}\right\}$ where $b_{j} \in Y$. Then $B_{j} \alpha^{2}=\left\{b_{j}\right\} \alpha$. Assume that $b_{j} \in A_{i_{0}}$ for some $i_{0}$, so $B_{j} \alpha^{2}=\left\{a_{i_{0}}\right\}$. Since $a_{i_{0}} \in X \alpha^{2}$ and $a_{i_{0}} \in Y \alpha$, we obtain $a_{i_{0}} \in X \alpha^{2} \cap Y \alpha$, so $a_{i_{0}}\left(\alpha^{2}\right)^{-1} \in \pi_{\alpha^{2}}(Y \alpha)=$ $\left\{A_{1}, \ldots, A_{n}\right\}$. Thus there is $A_{k} \in \pi_{\alpha^{2}}(Y \alpha)$ such that $A_{k} \alpha^{2}=\left\{a_{i_{0}}\right\}$. Hence $B_{j} \alpha^{2}$ $=\left\{a_{i_{0}}\right\}=A_{k} \alpha^{2}$ and therefore $B_{j} \cup A_{k} \subseteq a_{i_{0}}\left(\alpha^{2}\right)^{-1}$. That means $\pi_{\alpha^{2}}(Y) \neq \pi_{\alpha}(Y)$ which is a contradiction. Then $J=\emptyset$. So we can write

$$
\alpha=\left(\begin{array}{cccccc}
A_{1} & \ldots & A_{n} & C_{1} & \ldots & C_{t} \\
a_{1} & \ldots & a_{n} & c_{1} & \ldots & c_{t}
\end{array}\right)
$$

where $A_{i} \cap Y \neq \emptyset, C_{k} \subseteq X \backslash Y ; a_{i} \in Y, c_{k} \in X \backslash Y$ for all $i=1, \ldots, n$ and $k=1, \ldots, t$. Hence $Y^{\prime}=\left\{a_{1}, \ldots, a_{n}\right\}$ and $X^{\prime}=\left\{c_{1}, \ldots, c_{t}\right\}$. Therefore, $(X \backslash Y) \alpha \subseteq$ $(X \backslash Y) \cup Y^{\prime}$.

(2) We have $X^{\prime}=\left\{c_{1}, \ldots, c_{t}\right\} \subseteq X \backslash Y$ and $X \backslash Y^{\prime} \alpha^{-1}=C_{1} \cup \ldots \cup C_{t}$. Suppose that there is $c_{p} \in X^{\prime} \cap Y^{\prime} \alpha^{-1}$. Then $c_{p} \in A_{i_{0}}$ for some $i_{0}$. So $C_{p} \alpha^{2}=\left\{a_{i_{0}}\right\}$. Since $a_{i_{0}} \in X \alpha^{2}$ and $a_{i_{0}} \in Y \alpha$, we obtain $a_{i_{0}} \in X \alpha^{2} \cap Y \alpha$, so $a_{i_{0}}\left(\alpha^{2}\right)^{-1} \in \pi_{\alpha^{2}}(Y \alpha)=$ 
$\left\{A_{1}, \ldots, A_{n}\right\}$. Thus there is $A_{k} \in \pi_{\alpha^{2}}(Y \alpha)$ such that $A_{k} \alpha^{2}=\left\{a_{i_{0}}\right\}$. Hence $C_{p} \alpha^{2}=\left\{a_{i_{0}}\right\}=A_{k} \alpha^{2}$ and therefore $C_{p} \cup A_{k} \subseteq a_{i_{0}}\left(\alpha^{2}\right)^{-1}$, which implies that $\pi_{\alpha^{2}}(Y) \neq \pi_{\alpha}(Y)$, a contradiction. So $X^{\prime} \cap Y^{\prime} \alpha^{-1}=\emptyset$. Therefore, $X^{\prime} \subseteq$ $X \backslash Y^{\prime} \alpha^{-1}$.

(3) Assume that $\left|y^{\prime} \alpha^{-1} \cap Y^{\prime}\right| \neq 1$ for some $y^{\prime} \in Y^{\prime}$. Suppose that there are $a_{i}$ and $a_{j}$ both belong to $A_{l}$ for some $l \in\{1, \ldots, n\}$ where $a_{i} \neq a_{j} \in Y^{\prime}$. Then $A_{i} \alpha^{2}=\left\{a_{l}\right\}=A_{j} \alpha^{2}$. Thus $A_{i} \cup A_{j} \subseteq a_{l}\left(\alpha^{2}\right)^{-1}$. Since $a_{l} \in X \alpha^{2} \cap Y$, we obtain $a_{l}\left(\alpha^{2}\right)^{-1} \in \pi_{\alpha^{2}}(Y)$, so $\left|\pi_{\alpha^{2}}(Y)\right| \leq n-1$ where $\left|\pi_{\alpha}(Y)\right|=n$. Thus $\pi_{\alpha}(Y) \neq \pi_{\alpha^{2}}(Y)$, which is a contradiction. That means $a_{i}$ and $a_{j}$ belong to different $A_{l}$ for all $i \neq j$. Hence $Y^{\prime} \cap A_{l} \neq \emptyset$ for all $l$ since $\left|Y^{\prime}\right|=n$ is finite. Therefore, $\left|y^{\prime} \alpha^{-1} \cap Y^{\prime}\right|=1$ for all $y^{\prime} \in Y^{\prime}$.

(4) Assume that $\left|x^{\prime} \alpha^{-1} \cap X^{\prime}\right| \neq 1$ for some $x^{\prime} \in X^{\prime}$. Suppose that there are $c_{u}$ and $c_{v} \in C_{k}$ for some $k \in\{1, \ldots, t\}$ where $c_{u} \neq c_{v} \in X^{\prime}$. Then $C_{u} \alpha^{2}=\left\{c_{k}\right\}$ $=C_{v} \alpha^{2}$. So $C_{u} \cup C_{v} \subseteq c_{k}\left(\alpha^{2}\right)^{-1}$. This implies that $\left|\pi_{\alpha^{2}}\right| \leq n+(t-1)<\left|\pi_{\alpha}\right|$ which is a contradiction. Thus $c_{u}$ and $c_{v}$ belong to different $C_{k}$ for all $u \neq v$. And $X^{\prime} \cap C_{k} \neq \emptyset$ for all $k$ since $\left|X^{\prime}\right|=t$ is finite. Therefore, $\left|x^{\prime} \alpha^{-1} \cap X^{\prime}\right|=1$ for all $x^{\prime} \in X^{\prime}$.

Corollary 3.6. Let $\alpha \in S(X, Y)$ be such that $X \alpha$ is finite. If $\alpha$ is left regular, then $\alpha$ is regular.

Proof. Assume that $\alpha$ is left regular. Then $X \alpha=X \alpha^{2}$ and $Y \alpha=Y \alpha^{2}$. Since $Y \alpha \subseteq Y$ and $Y \alpha \subseteq X \alpha$, we obtain $Y \alpha \subseteq X \alpha \cap Y$. Now, we prove $X \alpha \cap Y \subseteq Y \alpha$ by letting $y \in X \alpha \cap Y$. If $y \notin Y \alpha$, then $y \in(X \backslash Y) \alpha \subseteq$ $(X \backslash Y) \cup Y^{\prime}$ by Lemma 3.3(1). Thus $y \in X \backslash Y$ or $y \in Y^{\prime}$. Since $y \in Y$, it follows that $y \in Y^{\prime}=Y \alpha$ which is a contradiction. Therefore, $y \in Y \alpha$ and that $X \alpha \cap Y \subseteq Y \alpha$. So $X \alpha \cap Y=Y \alpha$ which implies that $\alpha$ is regular by Theorem 2.1 .

Corollary 3.7. Let $\alpha \in S(X, Y)$ be such that $\pi_{\alpha}$ is finite. If $\alpha$ is right regular, then $\alpha$ is regular.

Proof. Assume that $\alpha$ is right regular. Then $\pi_{\alpha}=\pi_{\alpha^{2}}$ is finite and $\pi_{\alpha}(Y)=$ $\pi_{\alpha^{2}}(Y)$. By using the same proof as given for Corollary 3.6 and Lemma 3.5(1), we obtain $X \alpha \cap Y=Y \alpha$ and hence $\alpha$ is regular as required.

Theorem 3.8. Let $\alpha \in S(X, Y)$ be such that $X \alpha$ is finite. Then $\alpha$ is left regular if and only if $\alpha$ is right regular. 
Proof. Assume that $\alpha$ is left regular. Then $X \alpha=X \alpha^{2}$ and $Y \alpha=Y \alpha^{2}$. Since $X \alpha$ is finite, we may write $X \alpha=\left\{a_{1}, \ldots, a_{n}, c_{1}, \ldots\right.$, $\left.c_{t}\right\}$ where $Y^{\prime}=\left\{a_{1}, \ldots, a_{n}\right\}$. By Lemma 3.3(1), we can write

$$
\alpha=\left(\begin{array}{cccccc}
A_{1} & \ldots & A_{n} & C_{1} & \ldots & C_{t} \\
a_{1} & \ldots & a_{n} & c_{1} & \ldots & c_{t}
\end{array}\right)
$$

where $A_{i} \cap Y \neq \emptyset, C_{k} \subseteq X \backslash Y ; a_{i} \in Y, c_{k} \in X \backslash Y$ for all $i=1, \ldots, n$ and $k=1, \ldots, t$. Since $\left|y^{\prime} \alpha^{-1} \cap Y^{\prime}\right|=\left|A_{i} \cap\left\{a_{1}, \ldots, a_{n}\right\}\right|=1$ for all $i=1, \ldots, n$, there is a permutation $\sigma$ on the set $\{1, \ldots, n\}$ such that $a_{i} \in A_{i \sigma}$ for all $i$. So we obtain $A_{i} \alpha^{2}=\left\{a_{i \sigma}\right\}$. Similarly, since $\left|x^{\prime} \alpha^{-1} \cap X^{\prime}\right|=\left|C_{k} \cap\left\{c_{1}, \ldots, c_{t}\right\}\right|=1$ for all $k=1, \ldots, t$, there is a permutation $\delta$ on the set $\{1, \ldots, t\}$ such that $c_{k} \in C_{k \delta}$ for all $k$. Thus $C_{k} \alpha^{2}=\left\{c_{k \delta}\right\}$. So

$$
\alpha^{2}=\left(\begin{array}{cccccc}
A_{1} & \ldots & A_{n} & C_{1} & \ldots & C_{t} \\
a_{1 \sigma} & \ldots & a_{n \sigma} & c_{1 \delta} & \ldots & c_{t \delta}
\end{array}\right) .
$$

That is $\pi_{\alpha}(Y)=\left\{A_{1}, \ldots, A_{n}\right\}=\pi_{\alpha^{2}}(Y)$ and $\pi_{\alpha}=\left\{A_{1}, \ldots, A_{n}, C_{1}, \ldots\right.$, $\left.C_{t}\right\}=\pi_{\alpha^{2}}$. Hence $\alpha$ is right regular.

Conversely, assume that $\alpha$ is right regular. Then $\pi_{\alpha}=\pi_{\alpha^{2}}$ and $\pi_{\alpha}(Y)=$ $\pi_{\alpha^{2}}(Y)$. Since $X \alpha$ is finite, we obtain $\pi_{\alpha}=\pi_{\alpha^{2}}$ is finite. Then by Lemma $3.5(1)$, we can write

$$
\alpha=\left(\begin{array}{cccccc}
A_{1} & \ldots & A_{n} & C_{1} & \ldots & C_{t} \\
a_{1} & \ldots & a_{n} & c_{1} & \ldots & c_{t}
\end{array}\right)
$$

where $A_{i} \cap Y \neq \emptyset, C_{k} \subseteq X \backslash Y ; a_{i} \in Y, c_{k} \in X \backslash Y$ for all $i=1, \ldots, n$ and $k=1, \ldots, t$. Since $\left|y^{\prime} \alpha^{-1} \cap Y^{\prime}\right|=1$ for all $y^{\prime} \in Y^{\prime}$ and $\left|x^{\prime} \alpha^{-1} \cap X^{\prime}\right|=1$ for all $x^{\prime} \in X^{\prime}$. So by the same proof as given above

$$
\alpha^{2}=\left(\begin{array}{cccccc}
A_{1} & \ldots & A_{n} & C_{1} & \ldots & C_{t} \\
a_{1 \sigma} & \ldots & a_{n \sigma} & c_{1 \delta} & \ldots & c_{t \delta}
\end{array}\right)
$$

where $\sigma$ is a permutation on the set $\{1, \ldots, n\}$ and $\delta$ is a permutation on the set $\{1, \ldots, t\}$. Thus $Y \alpha=\left\{a_{1}, \ldots, a_{n}\right\}=Y \alpha^{2}$ and $X \alpha=\left\{a_{1}, \ldots, a_{n}, c_{1}, \ldots, c_{t}\right\}=$ $X \alpha^{2}$. Hence $\alpha$ is left regular.

Theorem 3.9. Let $\alpha \in S(X, Y)$. Then the following statements are equivalent:

(1) $\alpha$ is intra-regular.

(2) $|X \alpha|=\left|X \alpha^{2}\right|,|Y \alpha|=\left|Y \alpha^{2}\right|$ and $|X \alpha \backslash Y|=\left|X \alpha^{2} \backslash Y\right|$.

(3) $\alpha^{2} \in J_{\alpha}$. 
Proof. It is clear that $(2) \Leftrightarrow(3)$ by Lemma 2.2(3). If $\alpha$ is intra-regular, then $\alpha=\beta \alpha^{2} \gamma$ for some $\beta, \gamma \in S(X, Y)$ and we have $\alpha^{2}=\alpha \cdot \alpha \cdot 1_{X}$, so $\alpha \mathcal{J} \alpha^{2}$ and that $\alpha^{2} \in J_{\alpha}$. Conversely, if $\alpha^{2} \in J_{\alpha}$, then $\alpha=\beta \alpha^{2} \gamma$ for some $\beta, \gamma \in S(X, Y)$. So $\alpha$ is intra-regular.

Since $L_{\alpha} \subseteq J_{\alpha}$ and $R_{\alpha} \subseteq J_{\alpha}$, the following corollary is a direct consequences of Theorem 3.1, Theorem 3.2 and Theorem 3.9.

Corollary 3.10. Let $\alpha \in S(X, Y)$. Then $\alpha$ is intra-regular if one of the following conditions holds.

(1) $\alpha$ is left regular.

(2) $\alpha$ is right regular.

Theorem 3.11. Let $\alpha \in S(X, Y)$ be such that $X \alpha$ is a finite set. Then the following statements are equivalent:

(1) $\alpha$ is left regular.

(2) $\alpha$ is right regular.

(3) $\alpha$ is intra-regular.

Proof. (1) $\Leftrightarrow$ (2) follows from Theorem 3.8. (2) $\Rightarrow$ (3) is Corollary 3.10(2). Now, assume that $\alpha$ is intra-regular. Then $|X \alpha|=\left|X \alpha^{2}\right|$ and $|Y \alpha|=\left|Y \alpha^{2}\right|$. Since $X \alpha^{2} \subseteq X \alpha,\left|X \alpha^{2}\right|=|X \alpha|$ and $X \alpha$ is finite, we obtain $X \alpha=X \alpha^{2}$. Similarly, we have $Y \alpha=Y \alpha^{2}$. Therefore, $\alpha$ is left regular by Theorem 3.1.

The following examples show that the finiteness of $X \alpha$ in Theorem 3.11 is necessary.

Example 2. Let $X$ be the set of all natural numbers and $Y$ the set of all positive even integers. Let $\alpha$ be defined by $n \alpha=n+4$ for all $n \in X$. Then $\alpha \in S(X, Y)$ and $n \alpha^{2}=n+8$ for all $n \in X$. Thus we have $|X \alpha|=\aleph_{0}=\left|X \alpha^{2}\right|$, $|Y \alpha|=\aleph_{0}=\left|Y \alpha^{2}\right|$ and $|X \alpha \backslash Y|=\aleph_{0}=\left|X \alpha^{2} \backslash Y\right|$. So $\alpha$ is intra-regular. But $\alpha$ is not left regular, since $Y \alpha=\{2 n+4\} \neq\{2 n+8\}=Y \alpha^{2}$.

Now, let $\beta$ be defined by

$$
n \beta= \begin{cases}n & , n \in\{1,2,3,5\} \\ 2 & , n=4 ; \\ n-2 & , \text { otherwise. }\end{cases}
$$


Then $\beta \in S(X, Y)$ and

$$
n \beta^{2}= \begin{cases}n & , n \in\{1,2,3,5\} \\ 2 & , n \in\{4,6\} \\ 5 & , n \in\{7,9\} \\ n-4 & , \text { otherwise }\end{cases}
$$

Thus we have $|X \beta|=\aleph_{0}=\left|X \beta^{2}\right|,|Y \beta|=\aleph_{0}=\left|Y \beta^{2}\right|$ and $|X \beta \backslash Y|=\aleph_{0}=$ $\left|X \beta^{2} \backslash Y\right|$. So $\beta$ is intra-regular. But $\beta$ is not right regular, since $\pi_{\beta}(Y)=$ $\{\{2,4\},\{2 n+4\}\} \neq\{\{2,4,6\},\{2 n+6\}\}=\pi_{\beta^{2}}(Y)$.

\section{The Number of Left Regular Elements}

Throughout this section, $X$ is a finite set with $n$ elements and $Y \subseteq X$ has $r$ elements. To count the number of left regular elements of $S(X, Y)$, we recall that the number of combinations of $n$ distinct things taken $r$ at a time written $\left(\begin{array}{l}n \\ r\end{array}\right)$ is given by $\left(\begin{array}{l}n \\ r\end{array}\right)=\frac{n !}{(n-r) ! r !}$. That is, $\left(\begin{array}{l}n \\ r\end{array}\right)$ is the number of ways that $r$ objects can be chosen from $n$ distinct objects.

Lemma 4.1. Let $\alpha \in S(X, Y)$ be a left regular element. Then $\left.\alpha\right|_{Y}: Y \rightarrow$ $Y$ has $\sum_{k=1}^{r}\left(\begin{array}{l}r \\ k\end{array}\right) k ! k^{r-k}$ forms.

Proof. Since $\alpha \in S(X, Y)$ is a left regular element, we obtain $X \alpha=X \alpha^{2}$ is finite and $Y \alpha=Y \alpha^{2}$. We have $\left.\alpha\right|_{Y}: Y \rightarrow Y$ since $Y \alpha \subseteq Y$. Suppose that $Y \alpha=Y^{\prime}$ has $k$ elements. Let $Y^{\prime}=\left\{y_{1}^{\prime}, \ldots, y_{k}^{\prime}\right\}$. Since $\alpha \in S(X, Y)$, by Lemma 3.3 we can write

$$
\left.\alpha\right|_{Y}=\left(\begin{array}{ccc}
B_{1} & \ldots & B_{k} \\
y_{1}^{\prime} & \ldots & y_{k}^{\prime}
\end{array}\right)
$$

where $\bigcup_{i=1}^{k} B_{i}=Y$ and $\left|B_{i} \cap Y^{\prime}\right|=1$ for all $i=1, \ldots, k$. So $\left.\alpha\right|_{Y^{\prime}}: Y^{\prime} \rightarrow Y^{\prime}$ is a permutation. Thus $\left.\alpha\right|_{Y^{\prime}}$ can have $k$ ! forms. Since $Y \alpha=Y^{\prime}$ and $\left|Y \backslash Y^{\prime}\right|=r-k$, the number of ways of placing $r-k$ distinct elements into $k$ distinct places is $k^{r-k}$. Hence $\left.\alpha\right|_{Y}$ can have $\left(\begin{array}{l}r \\ k\end{array}\right) k ! k^{r-k}$ forms. Since $1 \leq k \leq r$, the number of the maps $\left.\alpha\right|_{Y}$ when $\alpha$ is left regular is $\sum_{k=1}^{r}\left(\begin{array}{l}r \\ k\end{array}\right) k ! k^{r-k}$. 
Lemma 4.2. Let $\alpha \in S(X, Y)$ be a left regular element. If $X^{\prime} \neq \emptyset$, then $\left.\alpha\right|_{X^{\prime}}: X^{\prime} \rightarrow X^{\prime}$ has $\sum_{m=1}^{n-r}\left(\begin{array}{c}n-r \\ m\end{array}\right) m !$ forms.

Proof. Since $\alpha \in S(X, Y)$ is a left regular element, we have $\left|x^{\prime} \alpha^{-1} \cap X^{\prime}\right|=1$ for all $x^{\prime} \in X^{\prime}$ by Lemma 3.3(4). Then $\left.\alpha\right|_{X^{\prime}}: X^{\prime} \rightarrow X^{\prime}$ is a permutation. Suppose that $X^{\prime}$ has $m$ elements. Let $X^{\prime}=\left\{x_{1}^{\prime}, \ldots, x_{m}^{\prime}\right\}$ and $\delta$ is the permutation on the set $\{1, \ldots, m\}$. So we can write

$$
\left.\alpha\right|_{X^{\prime}}=\left(\begin{array}{ccc}
x_{1}^{\prime} & \ldots & x_{m}^{\prime} \\
x_{1 \delta}^{\prime} & \ldots & x_{m \delta}^{\prime}
\end{array}\right) .
$$

Thus $\left.\alpha\right|_{X^{\prime}}$ can have $m$ ! forms. Since $1 \leq m \leq n-r$, the number of the maps $\left.\alpha\right|_{X^{\prime}}$ when $\alpha$ is left regular is $\sum_{m=1}^{n-r}\left(\begin{array}{c}n-r \\ m\end{array}\right) m$ ! .

Theorem 4.3. The number of left regular elements in $S(X, Y)$ is

$$
\sum_{m=0}^{n-r} \sum_{k=1}^{r}\left(\begin{array}{l}
r \\
k
\end{array}\right) k ! k^{r-k}\left(\begin{array}{c}
n-r \\
m
\end{array}\right) m !(k+m)^{n-r-m}
$$

where $|X|=n,|Y|=r$.

Proof. Let $\alpha \in S(X, Y)$ be a left regular element. Suppose that $Y^{\prime}$ has $k$ elements and $X^{\prime}$ has $m$ elements. Let $Y^{\prime}=\left\{y_{1}^{\prime}, \ldots, y_{k}^{\prime}\right\}$ and $X^{\prime}=\left\{x_{1}^{\prime}, \ldots, x_{m}^{\prime}\right\}$. Then by Lemma 3.3, we can write

$$
\alpha=\left(\begin{array}{cccccc}
A_{1} & \ldots & A_{k} & C_{1} & \ldots & C_{m} \\
y_{1}^{\prime} & \ldots & y_{k}^{\prime} & x_{1}^{\prime} & \ldots & x_{m}^{\prime}
\end{array}\right)
$$

where $A_{i} \cap Y \neq \emptyset, C_{j} \subseteq X \backslash Y$ and $\left|A_{i} \cap Y^{\prime}\right|=1,\left|C_{j} \cap X^{\prime}\right|=1$ for all $i=1, \ldots, k$ and $j=1, \ldots, m$.

If $X^{\prime}=\emptyset$, then $\bigcup_{i=1}^{m} C_{i}=\emptyset$, that is $\alpha=\left(\begin{array}{ccc}A_{1} & \ldots & A_{k} \\ y_{1}^{\prime} & \ldots & y_{k}^{\prime}\end{array}\right)$. By Lemma 4.1, $\left.\alpha\right|_{Y}$ has $\sum_{k=1}^{r}\left(\begin{array}{l}r \\ k\end{array}\right) k ! k^{r-k}$ forms. Since the number of ways of placing $n-r$ elements of the set $X \backslash Y$ into $k$ distinct places is $k^{n-r}$, in this case the number of left regular elements is $\sum_{k=1}^{r}\left(\begin{array}{l}r \\ k\end{array}\right) k ! k^{n-k}$. 
If $X^{\prime} \neq \emptyset$, then by Lemma 4.1 we have the number of the maps $\left.\alpha\right|_{Y}$ when $\alpha$ is left regular is $\sum_{k=1}^{r}\left(\begin{array}{l}r \\ k\end{array}\right) k ! k^{r-k}$. And by Lemma 4.2 , the number of $\left.\alpha\right|_{X^{\prime}}$ is $\sum_{m=1}^{n-r}\left(\begin{array}{c}n-r \\ m\end{array}\right) m$ !. So, the number of $\left.\alpha\right|_{Y \cup X^{\prime}}$ is $\sum_{m=1}^{n-r} \sum_{k=1}^{r}\left(\begin{array}{l}r \\ k\end{array}\right) k ! k^{r-k}\left(\begin{array}{c}n-r \\ m\end{array}\right) m !$. Now, $X \backslash\left(Y \cup X^{\prime}\right)$ has $n-r-m$ elements, and there are $(k+m)^{n-r-m}$ ways to placing $n-r-m$ elements into $k+m$ places. Therefore, the number of left regular elements in this case is

$$
\sum_{m=1}^{n-r} \sum_{k=1}^{r}\left(\begin{array}{l}
r \\
k
\end{array}\right) k ! k^{r-k}\left(\begin{array}{c}
n-r \\
m
\end{array}\right) m !(k+m)^{n-r-m} .
$$

Observe that if we put $m=0$ in the above expression, then we obtain $\sum_{k=1}^{r}\left(\begin{array}{l}r \\ k\end{array}\right) k ! k^{n-k}$. Therefore, we conclude that the number of left regular elements in $S(X, Y)$ is

$$
\sum_{m=0}^{n-r} \sum_{k=1}^{r}\left(\begin{array}{l}
r \\
k
\end{array}\right) k ! k^{r-k}\left(\begin{array}{c}
n-r \\
m
\end{array}\right) m !(k+m)^{n-r-m} .
$$

Remark. Since left regular, right regular and intra-regular elements of $S(X, Y)$ are the same when $X$ is a finite set, we obtain the numbers of left regular, right regular and intra-regular elements are equal.

\section{Acknowledgments}

This research was supported by Chiang Mai University.

\section{References}

[1] A.H. Clifford, G.B. Preston, The Algebraic Theory of Semigroups, Mathematical Surveys, No.7, vol.1 and 2, American Mathematical Society, Providence, RI, USA (1961) and (1967).

[2] K.D. Magill, Jr., Subsemigroups of $S(X)$, Math. Japon., 11 (1966), 109115. 
[3] P. Honyam, J. Sanwong, Semigroup of transformations with invariant set, J. Korean Math. Soc., 48, No. 2 (2011), 289-300.

[4] S. Nenthein, P. Youngkhong, Y. Kemprasit, Regular elements of some transformation semigroup, PU.M.A., 16, No. 3 (2005), 307-314. 